International Journal of Pure and Applied Mathematics

Volume 102 No. 3 2015, 447-472

ISSN: 1311-8080 (printed version); ISSN: 1314-3395 (on-line version)

url: http://www.ijpam.eu

doi: http://dx.doi.org/10.12732/ijpam.v102i3.4

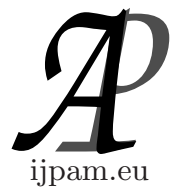

\title{
A STAGE STRUCTURED ECO-EPIDEMIOLOGICAL MODEL WITH DISEASE IN THE PREY AND IMPULSIVE EFFECTS
}

\author{
Shashi Kant ${ }^{1 \S}$, Vivek Kumar ${ }^{2}$ \\ 1,2 Department of Applied Mathematics \\ Delhi Technological University \\ Delhi, 110042, INDIA
}

\begin{abstract}
This paper aims to develop a new type of stage-structured ecoepidemiological model with disease in the prey and impulsive effects. We are dealing the interaction among five type of populations viz. juvenile prey, adult prey, diseased prey, juvenile predator and adult predator. We proved positivity and boundedness of the system. Analysis in terms of global attractivity, uniform persistence has been carried out. Finally, a pest control strategy is proposed.
\end{abstract}

AMS Subject Classification: 34XX, 34C23, 34C25, 34C28

Key Words: stage-structured population, global attractivity, pest control strategy

\section{Introduction}

The goal in this paper is to study a stage-structured predator-prey model with linear functional response with disease in the prey population and impulsive effects. It is a great concern of researchers in prey-predator relationship to understand the predator's functional response (predator's feeding rate upon prey), in other words the rate of prey consumption by an average predator. In literature there have been proposed several functional responses. Some of them are listed here for reference: $g(x)=C(t) x$ : Holling type I or linear function response,

Received: January 10, 2015

(C) 2015 Academic Publications, Ltd.

$\S$ Correspondence author url: www.acadpubl.eu 
$g(x)=\frac{C(t) x}{m+x}$ : Holling type II, $g(x)=\frac{C(t) x^{p}}{1+m x^{p}}, 0<p \leq 1$ : Generalized type II Holling functional response, $g(x)=\frac{C(t) x^{2}}{m+x^{2}}$ : Holling type III, $g(x)=\frac{C(t) x}{a+x+\frac{x^{2}}{m}}$ : Holling type IV, $g(x, y)=\frac{x y}{a y+x}$ : Ratio dependent, $g(x, y)=\frac{C x y}{(1+a x)(1+b y)}$ : Crowley-Martin type functional response, Hassell-Verley type functional response, $g(x, y)=\frac{C x}{1+k_{1} x+k_{2} y}$ :Beddington-De Anglis type, Function response of the type $g(x)=k\left(1-e^{-C x}\right)$ [Ivlev]. Many papers are available in literature for these functional responses. It is also remarkable that Holling type functional responses are more frequently used as compare to other functional responses. However, linear or volterra type functional responses is also frequent. Motivated by [7], in the present study we consider linear functional response.

Impulsive differential equations (IDE) are suitable to study the evolution process. IDE have been widely used in the population dynamics, pest control etc. In recent decades researchers introduced the concept of time delay. No dought this increases the complexity of the system but at the same this provides a new approach of thinking. Many mathematical models has been published by using the impulsive effects and delay simultaneously, for reference we may refer readers to the following mathematical models (and references therein):

- Zhongui Xiang (2010) [35]

$$
\left\{\begin{array}{l}
\dot{x}(t)=r x(t)\left(1-\frac{x(t)}{k}\right)-\frac{\beta x(t) y_{2}(t)}{1+\alpha x(t)} \\
y_{1}^{\prime}(t)=\frac{k \beta x(t) y_{2}(t)}{1+\alpha x(t)}-\frac{e^{-\omega \tau} k \beta x(t-\tau) y_{2}(t-\tau)}{1+\alpha x(t-\tau)}-\omega y_{1}(t) \\
y_{2}^{\prime}(t)=\frac{e^{-\omega \tau} k \beta x(t-\tau) y_{2}(t-\tau)}{1+\alpha x(t-\tau)}-\omega y_{2}(t)-\mu y_{2}^{2}(t) \\
\triangle x(t)=-p x(t), \triangle y_{1}=0, \triangle y_{2}=0
\end{array}\right.
$$

- Xinyu Song et al (2009) [32]

$$
\left\{\begin{array}{l}
\dot{x}=x(t)\left[r_{1}-a_{11} x(t)-a_{12} y_{2}(t)\right], t \neq n T \\
\dot{y_{1}}=\alpha x(t) y_{2}(t)-\gamma y_{1}(t)-\alpha e^{-\gamma \tau} x(t-\tau) y_{2}(t-\tau), t \neq n T \\
\dot{y_{2}}=-a_{22} y_{2}^{2}(t)-\gamma_{2} y_{2}(t)+\alpha e^{-\gamma \tau} x(t-\tau) y_{2}(t-\tau), t \neq n T \\
x\left(t^{+}\right)=(1-\delta) x(t), y_{1}\left(t^{+}\right)=y_{1}(t), y_{2}\left(t^{+}\right)=y_{2}(t), t=n T .
\end{array}\right.
$$

- Jian-Jun-Jia et al (2008)[9]

$$
\left\{\begin{array}{l}
x_{1}^{\prime}(t)=x_{1}(t)\left(a-b x_{1}(t)\right)-\beta x_{1}(t) x_{3}(t), t \neq n \tau \\
x_{2}^{\prime}(t)=\beta k x_{1}(t) x_{3}(t)-e^{-\omega \tau_{1}} \beta k x_{1}\left(t-\tau_{1}\right) x_{3}\left(t-\tau_{1}\right)-\omega x_{2}(t), t \neq n \tau \\
x_{3}^{\prime}(t)=e^{-\omega \tau_{1} \omega} \beta k x_{1}\left(t-\tau_{1}\right) x_{3}\left(t-\tau_{1}\right)-d_{3} x_{3}(t)-E x_{3}(t), t \neq n \tau \\
\triangle x_{1}(t)=\mu, \triangle x_{2}(t)=0, \triangle x_{3}(t)=0, t=n \tau
\end{array}\right.
$$


For more detailed dynamics of the models (1.1), (1.2) and (1.3), it is advisable to refer to $[35,32,9]$. Each living specie (including human) grows through different stages of age. Three main stages may be immature (juvenile), young age and old age. Broadly speaking we may categorize into two stages namely immature and mature stages. Mature stage may include young age and old age. Durations of immature and mature may vary for species. Age and sex wise data for human population may be found from population census reports. To the best of my knowledge, the age-wise data for other living species is not readily available. Therefore, there is a urgent need of such data for further research. In India, some of the livestock quinquennial censuses are being conducted e.g. tiger census etc. Such type of censuses are very important because from this we may have some idea about the bio-diversity, niche, endangered species etc. Unfortunately such censuses are not so frequent in the world. However, in published literature, theoretical models with stage structure for prey and/or predator are available. Some of them are cited here to understand this topic better. Some prey structured models are:

- Linfei Nie and Zhidong Ting (2013)[12], Xu et al (2004) [29] proposed the following ratio dependent model with stage structured of prey:

$$
\left\{\begin{array}{l}
\frac{d x_{1}}{d t}=a x_{2}-r_{1} x_{1}-b x_{1}, \\
\frac{d x_{2}}{d t}=b x_{1}-b_{1} x_{2}^{2}-\frac{a_{1} x_{2} x_{3}}{m x_{3}+x_{2}}, \\
\frac{d x_{3}}{d t}=-r x_{3}+\frac{a_{2} x_{2} x_{3}}{m x_{3}+x_{2}} .
\end{array}\right.
$$

- Chao Liu et al (2009) [3] proposed the prey structured model

$$
\left\{\begin{array}{l}
\dot{x_{1}}=r_{1} x_{2}-d_{1} x_{1}-\alpha x_{1}-S_{1} x_{1}^{2}-\beta x_{1} y \\
\dot{x_{2}}=\alpha x_{1}-d_{2} x_{2} \\
\dot{y}=\beta x_{1}(t-\tau) y(t-\tau)-d_{3} y-S_{2} y^{2}-E(t) y(t), \\
0=E(t)(w y(t)-c)-r .
\end{array}\right.
$$

Some predator structured models are cited here:

- Shengqiung Liu and Edoardo Beretta(2006) [24]:

$$
\left\{\begin{array}{l}
\frac{d x}{d t}=r x(t)\left(1-\frac{x(t)}{k}\right)-\frac{b x(t) y(t)}{1+k_{1} x(t)+k_{2} y(t)}, \\
\frac{d y(t)}{d t}=\frac{\eta b e^{-d_{j} \tau} x(t-\tau) y(t-\tau)}{1+k_{1} x(t-\tau)+k_{2} y(t-\tau)}-d y(t), \\
\frac{d y_{j}(t)}{d t}=\frac{\eta b x(t) y(t)}{1+k_{1} x(t)+k_{2} y(t)}-\frac{\eta e^{-d_{j} \tau} x(t-\tau) y(t-\tau)}{1+k_{1} x(t-\tau)+k_{2} y(t-\tau)}-d_{j} y_{j}(t) .
\end{array}\right.
$$


- Paul Georgescu and Ying-Hen Hsieh(2007) [19]:

$$
\left\{\begin{array}{l}
\frac{d x}{d t}=x(t)(r-a x(t))-\frac{b x(t)}{1+m x(t)} y_{2} \\
\frac{d y_{1}}{d t}=\frac{k b x(t)}{1+m x(t)} y_{2}-\left(D+d_{1}\right) y_{1}(t) \\
\frac{d y_{2}}{d t}=D y_{1}-d_{2} y_{2}(t)
\end{array}\right.
$$

- Yonghui Xia, Jinde Cao Sui Sun Cheng(2007)[33] proposed predator structured using Holling type IV model:

$$
\left\{\begin{array}{l}
\frac{d x}{d t}=x(t)\left[r_{1}(t)-a_{1}(t) \int_{-\infty}^{t} k(t-s) x(s) d s-\frac{a_{2} y_{2}(t)}{\mathrm{R}^{\frac{x^{2}}{m}+x(t)+a}}\right] \\
\frac{d y_{1}}{d t}=\frac{b_{1}(t) x(t) y_{2}(t)}{\frac{x^{2}}{m}+x(t)+a}-\beta(t) y_{1}(t)-b_{1}(t-\tau) e^{-\left(\begin{array}{c}
\left.{ }_{t-\tau} \beta(s) d s\right) \\
\frac{\mathrm{R}_{t}(t-\tau)}{m}+x_{1}(t-\tau)+a
\end{array}\right.} \\
\frac{d y_{2}}{d t}=b_{1}(t-\tau) e^{\left.-{ }_{t-\tau} \beta(s) d s\right)} \frac{x(t-\tau) y_{2}(t-\tau)}{\frac{x^{2}(t-\tau)}{m}+x_{1}(t-\tau)+a}-r_{2} y_{2}(t) .
\end{array}\right.
$$

- Rui Xu et al(2004)[21] proposed the following predator structured model:

$$
\left\{\begin{array}{l}
\dot{x}(t)=x(t)\left(r(t)-a(t) x\left(t-\tau_{1}\right)-a_{1}(t) y_{2}(t)\right) \\
\dot{y}(t)=a_{2}(t) x\left(t-\tau_{2}\right) y_{2}\left(t-\tau_{2}\right)-r_{1}(t) y_{t}-b_{1} y_{1}(t) \\
\dot{y_{2}}(t)=-r_{2}(t) y_{2}(t)+b_{1} y_{1}(t)
\end{array}\right.
$$

for more references and detail on stage-structured models we may refer ([32, $31,30,29,21,12,9,3]$ and references cited therein) for further reading. In this paper, we consider two stages of age viz. immature and mature for both prey and predator population as mentioned earlier.

Heavy use of pesticides may cause serious problems in our society. As an example we can take the case of Punjab (India) where excess use of pesticides caused many cancer patients [18]. The reason is that use of pesticides kills the unwanted species but at the same time few traces left in food grains(crops). After consumption of this food by human caused morbidity and diseases e.g. cancer, gas problem, BP etc. Thus use of pesticides in grains (crops) is dangerous. But without use of pesticides good food production is not possible. Hence, we need to maintain balanced use of pesticides. In pest control problems mathematical modeling has an important role especially IDE as discussed in second paragraph above. In such type of problems our main target is to find the economic threshold level (ETL).

Prey-predator models with disease dynamics has also been emerged in recent decades. Such type of mathematical models are termed as eco-epidemiological 
models. Eco-epidemiological models get popularized by the most seminal work of Kermack-Mckendrick [10] and Volterra [27]. The work of [10, 27] have been cited in large number of papers. The elementary work in $[10,27]$ proved to be a mile stone in the area of eco-epidemiological models. After the work in $[10,27]$ many mathematical models with disease in prey and/or predators has been published. For instance, we write two such papers:

- Samanta(2010) [22] proposed the following model with disease in the prey population

$$
\left\{\begin{array}{l}
\frac{d x_{1}(t)}{d t}=x_{1}(t)\left[r(t)-k_{1}(t)\left(x_{1}(t)+x_{2}(t)\right)-a_{1}(t) x_{3}(t)-\beta(t) x_{2}(t)\right] \\
\frac{d x_{2}(t)}{d t}=x_{2}(t)\left[r(t)-k_{2}(t)\left(x_{1}(t)+x_{2}(t)\right)-a_{2}(t) x_{3}(t)+\beta(t) x_{1}(t)\right] \\
\frac{d x_{3}(t)}{d t}=-d(t) x_{3}(t)-b(t) x_{3}^{2}(t)+c_{1}(t) x_{3}(t-\tau) x_{1}(t-\tau) \\
+c_{2}(t) x_{3}(t-\tau) x_{2}(t-\tau) .
\end{array}\right.
$$

- G-P. Hu and X-L. Li (2012) [7] proposed the following model with disease in the prey population

$$
\left\{\begin{array}{l}
\frac{d S}{d t}=r S\left(1-\frac{S+I}{k}\right)-S I \beta-p_{1} S Y \\
\frac{d I}{d t}=-c I+S I \beta-p_{2} I Y \\
\frac{d Y}{d t}=-d Y+q p_{1} S(t-\tau) Y(t-\tau)+q p_{2} I(t-\tau) Y(t-\tau)
\end{array}\right.
$$

we may refer readers for more papers $([16,6,23,17,20,15,4,11,33]$ and references cited therein).

Motivated by the above discussion, we proposed a delayed eco-epidemiological model with stage structured for both prey and predator with disease in the prey population and impulsive effects. As promised in first paragraph we have considered linear functional response. All the parameters are time independent and positive.

Rest of the paper is structured as follows. Next section is dealing with model formulation. In Section 3, analysis of the model regarding global attractivity is presented. In Section 4, uniform persistence and pest control strategy is investigated. 


\section{Model Formulation}

Basic S-I model is

$$
\left\{\begin{array}{l}
\frac{d S}{d t}=b S(t)-\beta S(t) I(t)-d S(t), \\
\frac{d I}{d t}=\beta S(t) I(t)-d I(t)-r I(t),
\end{array}\right.
$$

where $\mathrm{S}(\mathrm{t})$ and $\mathrm{I}(\mathrm{t})$ denotes the susceptible and infected population respectively. Based on the basic S-I model (2.1), recently in [26], a high dimensional mathematical model is proposed and analyzed the following model:

$$
\begin{cases}\frac{d S_{j}(t)}{d t}=r S(t)-d_{1} S_{j}(t)-r e^{-d_{1} \tau} S(t-\tau), & t \neq(n+l-1) T, t \neq n T, \\ \frac{d S(t)}{d t}=r e^{-d_{1} \tau} S(t-\tau) & t \neq(n+l-1) T, t \neq n T, \\ -d_{2} S^{2}(t)-\beta S(t) I^{2}(t)-\frac{\alpha S(t) Y(t)}{1+\omega Y(t)}, & t \neq(n+l-1) T, t \neq n T, \\ \frac{d I(t)}{d t}=\beta S(t) I^{2}(t)-d_{3} I(t), & t \neq(n+l-1) T, t \neq n T, \\ \frac{d Y_{j}(t)}{d t}=\lambda \frac{\alpha S(t) y(t)}{1+\omega y(t)}-\left(m+d_{4}\right) Y_{j}(t), & t \neq(n+l-1) T, t \neq n T, \\ \frac{d Y(t)}{d t}=m Y_{j}(t)-d_{5} Y(t), & t=(n+l-1) T, \\ \Delta S_{j}(t)=0, \Delta S(t)=0, \Delta I(t)=0, & \\ \Delta Y_{j}(t)=q, \Delta Y(t)=0, & t=n T . \\ \Delta S_{j}(t)=0, \Delta S(t)=0, \Delta I(t)=p, & \\ \Delta Y_{j}(t)=0, \Delta Y(t)=0, & \end{cases}
$$

The detail of the model(2.2) can be found in [26]. Motivated by the above model [26], in the present study we propose the following mathematical model:

$$
\begin{cases}\frac{d S_{j}(t)}{d t}=r S(t)-d_{1} S_{j}(t)-r e^{-d_{1} \tau} S(t-\tau), & t \neq(n+l-1) T, t \neq n T, \\ \frac{d S(t)}{d t}=r e^{-d_{1} \tau} S(t-\tau)-d_{2} S^{2}(t) & \\ -\beta S(t) I^{2}(t)-p_{1} S(t) Y(t), & t \neq(n+l-1) T, t \neq n T, \\ \frac{d I(t)}{d t}=\beta S(t) I^{2}(t)-\left(d_{3}+d_{4}\right) I(t), & t \neq(n+l-1) T, t \neq n T, \\ \frac{d Y_{j}(t)}{d t}=q_{1} p_{1} S(t) Y(t)-\left(m+d_{5}\right) Y_{j}(t), & t \neq(n+l-1) T, t \neq n T, \\ \frac{d Y(t)}{d t}=m Y_{j}(t)-d_{6} Y(t), & t \neq(n+l-1) T, t \neq n T, \\ \Delta S_{j}(t)=0, \Delta S(t)=0, \Delta I(t)=0, & t=(n+l-1) T, \\ \Delta Y_{j}(t)=q, \Delta Y(t)=0, & \\ \Delta S_{j}(t)=0, \Delta S(t)=0, \Delta I(t)=p, & t=n T, \\ \Delta Y_{j}(t)=0, \Delta Y(t)=0, & \end{cases}
$$

where $S_{j}(t), S(t)$ and $I(t)$ denotes the immature, mature and infected pests (prey) respectively. $Y_{j}(t)$ and $Y(t)$ are immature and mature natural enemies 
(predator) respectively. $r$ is the intrinsic growth rate in absence of disease and predation. $d_{1}, d_{2}, d_{5}, d_{6}$ are natural death rates of immature,mature prey and predators respectively. The constants, $d_{3}$ and $d_{4}$ are natural death and death due to disease of infected pest respectively. $m$ is the number of immature predator which changes to mature predator. $p_{1}$ is predation rate and $q_{1}$ is the conversion rate at which ingested prey digested by predator. $\tau$ is the mean length of juvenile period of prey: $\mathrm{T}$ is the period of the impulsive effect. $\mathrm{q}$ is the released amount of the predator at every impulsive period $(n+l-1) T$ similarly $p$ is the released amount of the infective prey at every impulsive period nT. Keeping the point of biology and ecology in mind our proposed system is restricted to the region $\Omega=\left\{\left(S_{j}, S, I, Y_{j}, Y\right) \mid S_{j}, S, I, Y_{j}, Y \geq 0\right\}$. Let $C^{+}=$ $\left\{\phi=\left(\phi_{1}(s), \phi_{2}(s), \phi_{3}(s), \phi_{4}(s), \phi_{5}(s)\right) \in C: \phi_{i}>0(i=1,2,3,4,5)\right\}$, where $\phi_{i}(s)$ is non-negative, bounded and continuous function for $s \in[-\tau, 0]$. $C$ is the Banach Space $C\left([-\tau, 0], R^{5}\right)$ of continuous functions mapping the interval $[-\tau, 0]$ into $R^{5}$. We assume that our system satisfies the initial conditions:

$$
\phi \in C^{+} .
$$

For the continuity of initial conditions, we require

$$
\phi(0)=\int_{-\tau}^{0} r e^{d_{1} \mu} S(\mu) d \mu .
$$

It is observed that, the variable $S_{j}(t)$ representing immature pest population appears only in the first equation of our model, therefore we need to to consider the following subsystem of our model;

$$
\begin{cases}\frac{d S(t)}{d t}=r e^{-d_{1} \tau} S(t-\tau)-d_{2} S^{2}(t) & \\ -\beta S(t) I^{2}(t)-p_{1} S(t) Y(t), & t \neq(n+l-1) T, t \neq n T, \\ \frac{d I(t)}{d t}=\beta S(t) I^{2}(t)-\left(d_{3}+d_{4}\right) I(t), & t \neq(n+l-1) T, t \neq n T, \\ \frac{d Y_{(}(t)}{d t}=q_{1} p_{1} S(t) Y(t)-\left(m+d_{5}\right) Y_{j}(t), & t \neq(n+l-1) T, t \neq n T, \\ \frac{d Y(t)}{d t}=m Y_{j}(t)-d_{6} Y(t), & t \neq(n+l-1) T, t \neq n T, \\ \Delta S_{j}(t)=0, \Delta S(t)=0, \Delta I(t)=0, & \\ \Delta Y_{j}(t)=q, \Delta Y(t)=0, & t=(n+l-1) T, \\ \Delta S_{j}(t)=0, \Delta S(t)=0, \Delta I(t)=p, & t=n T . \\ \Delta Y_{j}(t)=0, \Delta Y(t)=0, & \end{cases}
$$

Lemma 1. Each Solution for system (2.3) with the given initial conditions (2.4) is strictly positive for all $t \geq 0$. 
Proof. First we observe that the solution of our model $(2.3), x(t)=$ $\left(S_{j}(t), S(t), I(t), Y_{j}(t), Y(t)\right)$ is a piecewise continous function on the interval $(n \tau,(n+1) \tau]$ and $x\left(n \tau^{+}\right)=\lim _{x \rightarrow n \tau^{+}} x(t)$. For ecological and biological point of view all the solution of our model should be strictly positive for all the time. In other words, we say that with positive initial value remain positive for all $t>0$.

Let us consider $S_{j}(t)>0, S(t)>0, I(t)>0, Y_{j}(t)>0, Y(t)>0$ for some time $t$, then we will show that the solutions of our main mathematical model $(2.3)\left(S_{j}(t), S(t), I(t), Y_{j}(t), Y(t)\right)$ are positive for all time $t>0$.

First of all, we will show that $S(t)>0$ for all $t>0$. Since we have considered that $S(t)>0$, there exists $t_{0}$ such that $S\left(t_{0}\right)=0$ then $t_{0}>0$. Let us assume that, $t_{0}$ is one such first time such that $S(t)=0$, that is $t_{0}=\inf \{t>0: S(t)=0\}$ then from first equation of our reduced system (2.6), we get the following:

$$
\dot{S}\left(t_{0}\right)=r e^{-d_{1} \tau} S(t-\tau)>0,
$$

hence for arbitrary sufficiently small $\varepsilon>0, \dot{S}\left(t_{0}-\varepsilon\right)>0$, but the definition of $t_{0}$ suggest that $S\left(t_{0}-\varepsilon\right) \leq 0$, which is a contradiction so we have proved that $S(t)>0 \forall t>0$.

Next, from the second equation of system (2.6), we have:

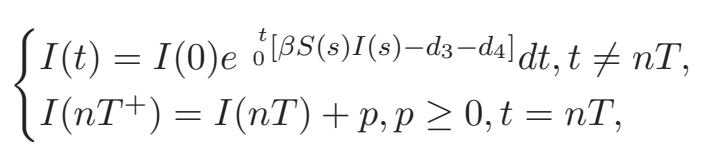

hence, it is obvious that $I(t)>0, \forall t>0$.

Next, from third equation of our system (2.6), we have,

$\frac{d Y_{j}(t)}{d t}=q_{1} p_{1} S(t) Y(t)-\left(m+d_{5}\right) Y_{j}(t)$,

$\Rightarrow \frac{d Y_{j}(t)}{d t}=\frac{q_{1} p_{1} S(t) Y(t) Y_{j}(t)}{Y_{j}(t)}-\left(m+d_{5}\right) Y_{j}(t)$,

$\Rightarrow \frac{d Y_{j}(t)}{Y_{j}(t)}=\left(\frac{q_{1} p_{1} S(t) Y(t)}{Y_{j}(t)}-\left(m+d_{5}\right)\right) d t$,

integrating it, we obtained,

$$
\left\{\begin{array}{l}
Y_{j}(t)=Y_{j}(0) e^{\mathrm{R}_{t}\left(\frac{q_{1} p_{1} S(t) Y(t)}{Y_{j}(t)}-\left(m+d_{5}\right)\right) d t} \\
Y_{j}\left(n T^{+}\right)=Y_{j}(n T)+q, q \geq 0
\end{array}\right.
$$

thus we have $Y_{j}(t) \geq 0 \forall t>0$.

Similarly, from the fourth equation of system (2.6), we have,

$$
\begin{aligned}
& \frac{d Y(t)}{d t}=m Y_{j}(t)-d_{6} Y(t), \\
& \Rightarrow \frac{d Y(t)}{d t}=\frac{m Y_{j}(t) Y(t)}{Y(t)}-d_{6} Y(t),
\end{aligned}
$$


$\Rightarrow \frac{d Y(t)}{Y(t)}=\left[\frac{m Y_{j}(t)}{Y(t)}-d_{6}\right] d t$

integrating it we have

$$
Y(t)=Y(0) e^{\mathrm{R}_{t}\left[\frac{m Y_{j}(t)}{Y(t)}-d_{6}\right] d t},
$$

thus we have $Y(t)>0, \forall t>0$. We have proved, $S(t)>0, I(t)>0, Y_{j}(t)>$ $0, Y(t)>0 \forall t>0$. Now we need to prove that $S_{j}(t)>0 \forall t>0$. For this, we consider the following equation:

$$
\dot{u}(t)=-r e^{-d_{1} \tau} S(t-\tau)-d_{1} u(t),
$$

and comparing with first equation of main system (2.3), we observe that if $\mathrm{u}(\mathrm{t})$ is the solution of above equation and if $S_{j}(t)>0$ satisfied the first equation of main system $(2.3)$, then $S_{j}(t)>u(t)$ for $0 \leq t \leq \tau$. From above equation, we have

$$
u(t)=e^{-d_{1} \tau}\left(S_{j}(0)-\int_{0}^{t}\left[r e^{d_{1}(s-\tau)} S(s-\tau) d s\right]\right),
$$

from continuity condition (2.5), we have,

$$
u(t)=e^{-d_{1} \tau}\left(\int_{-\tau}^{0}\left[r e^{d_{1} s} S(s) d s-\int_{0}^{\tau}\left[r e^{d_{1}(s-\tau)} S(s-\tau) d s\right]\right),\right.
$$

we notice that

$$
\int_{-\tau}^{0} r e^{d_{1} s} S(s) d s=\int_{0}^{\tau} r e^{d_{1}(s-\tau)} S(s-\tau) d s
$$

hence $u(t)=0 \Rightarrow S_{j}(t)>0$, since $u(t)$ is strictly decreasing so $S_{j}(t)>0, u(t)>$ 0 for $t \in[0, \tau]$, therefore $S_{j}(t)>0$ for $t \in[0, \tau]$. Now by induction method, we will show that $S_{j}(t)>0$ for $t \in[n \tau,(n+l) \tau], n=0,1,2,3, \ldots \ldots$. We have proved $S_{j}(t)>0, u(t)>0$ for $t \in[0, \tau]$ for $n=0$. Now we assume that $S_{j}(t)>0, u(t)>0$ for $t \in[0, \tau]$ is true for $n=0,1,2,3, \ldots \ldots k$, then $S_{j}(k \tau)>0$ for $n=k$. Consider the equation,

$$
\dot{u}(t)=-r e^{-d_{1} \tau} S(t-\tau)-d_{1} u(t), u(k \tau)=S_{j}(k \tau),
$$

from first equation of main system (2.3), we have $S_{j}(t)>u(t)$ for $t>k \tau$. Now the above equation is equivalent to

$$
u(t)=e^{-d_{1}(\tau-k \tau)}\left(S_{j}(k \tau)-\int_{k \tau}^{t}\left[r e^{d_{1}(s-\tau-k \tau)} S(s-\tau) d s\right]\right),
$$


hence

$$
u((k+1) \tau)=e^{-d_{1} \tau}\left(S_{j}(k \tau)-\int_{k \tau}^{(k+1) \tau}\left[r e^{d_{1}(s-(k+1) \tau)} S(s-\tau) d s\right]\right) .
$$

Since $\phi(0)=\int_{-\tau}^{0} r e^{d_{1} s} S(s) d s$, we have

$$
\phi_{(k \tau)}=\int_{k \tau}^{(k+1) \tau}\left[r e^{d_{1}(s-(k+1) \tau)} S(s-\tau) d s=\int_{(k-1) \tau}^{k \tau}\left[r e^{d_{1}(s-k \tau)} S(s) d s .\right.\right.
$$

So we obtain, $u((k+1) \tau)=0$ therefore $S_{j}(\tau)>0$. Hence, $S_{j}((k+1) \tau)>0$. From the above discussion we can conclude by induction that $S_{j}(t)>0 \forall t>0$.

Lemma 2. Solutions of system (2.3) are bounded.

Proof. Before proceeding the boundedness, let us introduce some sets and definitions. $R_{+}=[0, \infty), R_{+}^{5}=\left\{x \in R^{5}: x \geq 0, x=\left(S_{j}, S, I, Y_{j}, Y\right)\right\}$. $f=\left(f_{1}, f_{2}, f_{3}, f_{4}, f_{5}\right)$ be the map defined by the right hand side of the interior of the five equations of our main mathematical model () and let $N$ be the set of all non-negative integers. Let $V: R_{+} \times R_{+}^{5} \rightarrow R_{+}$, then $\mathrm{V}$ is said to belongs to a class $V_{0}$ if the following conditions are satisfied.

- $V$ is continuous in $[(k-1) T, k T] \times R_{+}^{5}, k \in N$ and for each $x \in R_{+}^{5}$ the following limits exists: $\lim _{(t, z) \rightarrow\left((k-1) T^{+}, x\right)} V(t, z)=V((k-1) T, x)$, $\lim _{(t, z) \rightarrow\left(k T^{+}, x\right)} V(t, z)=V\left(k T^{+}, x\right)$,

- $\mathrm{V}$ is locally Lipschtizian in $x$.

Definition 1. [26] Let $V \in V_{0}$, then for $(t, x) \in[(k-1) T, k T] \times R_{+}^{5}, k \in N$, the upper right derivative of $V(t, x)$ with respect to the impulsive differential equation system is defined as

$$
D^{+} V(t, x)=\lim _{h \rightarrow 0^{+}} \sup \frac{1}{h}[V(t+h, x+h f(t, x))-V(t, x)] .
$$

Now let us proceed for boundedness. Let

$$
V(t)=q_{1}\left(S_{j}(t)+S(t)+I(t)+Y_{j}(t)+Y(t)\right) .
$$

For $t \neq(n+l-1) T, t \neq n T$

$\Rightarrow D^{+} V(t)=r q_{1} S(t)-d_{2} q_{1} S^{2}(t)-d_{1} q_{1} S_{j}(t)-\left(d_{3}+d_{4}\right) q_{1} I(t)-d_{5} Y_{j}(t)-d_{6} Y(t)$.

Let $d=\min \left\{d_{1}, d_{3}, d_{4}, d_{5}, d_{6}\right\}, Q=\frac{q_{1}(r+d)^{2}}{4 d_{2}}$, we have 
$D^{+} V(t)<q_{1}\left((r+d) S(t)-d_{2} S^{2}(t)\right)-d V(t)<Q-d V(t)$. Thus we have the following impulsive differential inequalities;

$$
\left\{\begin{array}{l}
D^{+} V(t)<Q-d V(t), t \neq(n+l-1) T, t \neq n T, \\
V\left((n+l-1) T^{+}\right)=V((n+l-1) T)+q, t=(n+l-1) T, \\
V\left(n T^{+}\right)=V(n T)+q_{1} p, t=n T .
\end{array}\right.
$$

According to impulsive differential inequality theory, we have

$V(t) \leq V(0) e^{-d t}+\int_{0}^{t} Q e^{-d(t-s)} d s+\Sigma_{0<(n+l-1) T<n T<t}\left(q e^{-d(t-(n+l-1) T)}+\right.$ $\left.q_{1} p e^{-d(t-n T)}\right)$

$t \rightarrow \infty \Rightarrow$

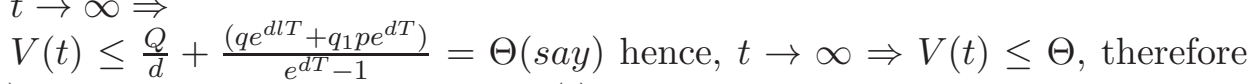
$V(t)$ is bounded. The definition of $V(t)$ proved that each solution of our system (2.3) is bounded. This completes the proof.

\section{Analysis of the Model}

We have the following lemmas from published literature for further use in the analysis.

Lemma 3. $[2,13,26]$ Let $V: R_{+} \times R_{+}^{3} \longrightarrow R_{+}$, and $V \in V_{0}$. Assume that

$$
\left\{\begin{array}{l}
D^{+} V(t, z(t)) \leq(\geq) g(t, V(t, z(t))), t \neq n \tau \\
V\left(t, z(t)^{+}\right) \leq(\geq) \Psi_{n}(V(t, z(t))), t=n \tau
\end{array}\right.
$$

where $g: R_{+} \times R_{+} \longrightarrow R$ is continuous in the domain $(n \tau,(n+1) \tau] \times R_{+}$and for each $x \in R_{+}, n \in N, \lim _{(t, y) \rightarrow\left((n \tau)^{+}, x\right)} g(t, y)=g\left((n \tau)^{+}, x\right)$ exist: $\Psi_{n}: R_{+} \rightarrow$ $R_{+}$is non decreasing. Let $r(t)=r\left(t, 0, u_{0}\right)$ be the maximal (minimal) solution of the scalar impulsive differential equation

$$
\left\{\begin{array}{l}
\dot{u}=g(t, u), t \neq n \tau, \\
u\left(t^{+}\right)=\Psi_{n}(u(t)), t=n \tau .
\end{array}\right.
$$

on $[0, \infty)$. Then $V\left(0^{+}, z_{0}\right) \leq(\geq) u_{0}$ implies that $V(t, z(t)) \leq(\geq) r(t), t \geq$ 0 , where $z(t)=z\left(t, 0, z_{0}\right)$ is a solution of above system of scalar impulsive differential equation on $[0, \infty)$.

Lemma 4. $[32,26,22]$ Consider the following delay differential equation

$$
\frac{d x(t)}{d t}=a x(t-\tau)-b x(t)-c x^{2}(t)
$$

where $a, b, c, \tau$ are positive constants and $x(t)>0$ for $t \in[-\tau, 0]$. Then we have 
1. if $a<b, \lim _{t \rightarrow \infty} x(t)=0$,

2. if $a>b, \lim _{t \rightarrow \infty} x(t)=\frac{a-b}{c}$.

Lemma 5. [1, 26] Impulsive system

$$
\begin{gathered}
\frac{d v(t)}{d t}=-c v(t), t \neq n T, n \in N, \\
\Delta v(t)=\mu, t=n T, n \in N,
\end{gathered}
$$

has a unique positive T-periodic solution $v(t)^{*}=\frac{\mu e^{-c(t-n T)}}{1-e^{-c T}}$ for $t \in(n T,(n+$ 1)T], $n \in N, v^{*}(0)=\frac{\mu}{1-e^{-c T}}$. For each solution $v(t)$ we have $v(t) \rightarrow v^{*}(t)$ as $t \rightarrow \infty$.

Lemma 6. [36, 26] System of impulsive differential equation

$$
\begin{gathered}
\frac{d u(t)}{d t}=a v(t)-b u(t), t \neq n T, \\
\frac{d v(t)}{d t}=-c v(t), t \neq n T, \\
\Delta u(t)=0, \Delta v(t)=\mu, t=n T,
\end{gathered}
$$

has a unique positive T-periodic solution $\left(u^{*}(t), v^{*}(t)\right)$, which takes the form;

$$
\left\{\begin{array}{l}
u^{*}(t)=u^{*}(0) e^{-b(t-n T)}+\frac{a \mu\left(e^{-b(t-n T)}-e^{-c(t-n T)}\right)}{(c-b)\left(1-e^{-c T}\right)}, \\
v^{*}(t)=\frac{\mu e^{-c(t-n T)}}{\left(1-e^{-c T}\right)}, \\
u^{*}(0)=\frac{a \mu\left(e^{-b T}-e^{-c T}\right)}{(c-b)\left(1-e^{-b T}\right)\left(1-e^{-c T}\right)}, \\
v^{*}(0)=\frac{\mu}{\left(1-e^{-c T}\right)},
\end{array}\right.
$$

for $t \in(n T,(n+1) T]$ and $n \in N$, and satisfies $u(t) \rightarrow u^{*}(t)$ and $v(t) \rightarrow v^{*}(t)$ as $t \rightarrow \infty$.

\subsection{Global Attractivity of the Mature Prey (Pest)-Extinction Periodic Solution}

Firstly, we investigate the prey extinction solution of the prey population is entirely absent i.e. $S(t)=0, t \geq 0$. In this case, our system (2.6) reduced to:

$$
\left\{\begin{array}{l}
\frac{d I}{d t}=-\left(d_{3}+d_{4}\right) I(t), t \neq n T \\
\triangle I(t)=p, t=n T \\
\frac{d Y_{j}(t)}{d t}=-\left(m+d_{5}\right) Y_{j}(t), t \neq(n+l-1) T, t \neq n T \\
\frac{d Y(t)}{d t}=m Y_{j}(t)-d_{6} Y(t), t \neq(n+l-1) T, t \neq n T \\
\triangle Y_{j}(t)=q, \triangle y(t)=0, t=(n+l-1) T
\end{array}\right.
$$

then from direct application of lemmas (5) and (6), we have the following important theorem: 
Theorem 1. System (2.6) has a mature prey-extinction periodic solution $\left(0, I^{*}(t), Y_{j}^{*}(t), Y^{*}(t)\right)$ for $t \in((n+l-1) T,(n+l) T], n \in N$ where

$$
\left\{\begin{array}{l}
I^{*}(t)=\frac{p e^{-\left(d_{3}+d_{4}\right)(t-n T)}}{1-e^{-\left(d_{3}+d_{4}\right) T}} \\
I^{*}(0)=\frac{p}{1-e^{-\left(d_{3}+d_{4}\right) T}} \\
Y^{*}(t)=Y^{*}(0) e^{-d_{6}(t+(n+l-1) T)}+\frac{m q\left(e^{-d_{6}(t-(n+l-1) T)}-e^{-\left(m+d_{5}\right)(t-(n+l-1) T)}\right.}{\left(m+d_{5}-d_{6}\right)\left(1-e^{-\left(m+d_{5}\right) T}\right)} \\
Y_{j}^{*}(t)=\frac{q e^{-\left(m+d_{5}\right)(t-(n+l-1) T)}}{\left(1-e^{-\left(m+d_{5} T\right)}\right)}, \\
Y_{j}^{*}(0)=\frac{q}{1-e^{\left(m+d_{5}\right) T}}, \\
Y^{*}(0)=\frac{m q\left(e^{-d_{6} T}-e^{-\left(m+d_{5}\right) T}\right)}{\left(m+d_{5}-d_{6}\right)\left(1-e^{-d_{6} T}\right)\left(1-e^{-\left(m+d_{5}\right) T}\right)} .
\end{array}\right.
$$

Secondly, we investigate the global attractivity of the mature-prey extinction periodic solution. Let us adopt the following notations,

$$
\left\{\begin{array}{l}
A_{1}=\frac{p e^{-\left(d_{3}+d_{4}\right) T}}{1-e^{-\left(d_{3}+d_{4}\right) T}}, \\
A_{2}=\frac{m q\left(e^{-d_{6} T}-e^{-\left(m+d_{5}\right) T}\right)}{\left(m+d_{5}-d_{6}\right)\left(1-e^{-d_{6} T}\right)\left(1-e^{-\left(m+d_{5}\right) T}\right)}, \\
B=\frac{m q\left(1-\frac{d_{6}}{\left(m+d_{5}\right)}\right)\left(\frac{d_{6}}{\left(m+d_{5}\right)}\left(1-e^{-\left(m+d_{5}\right) T}\right)^{\left(m+d_{5}-d_{6}\right)}\right)}{\left(m+d_{5}-d_{6}\right)\left(1-e^{-d_{6} T}\right)^{\frac{m+d_{5}}{\left(m+d_{5}-d_{6}\right)}}}, \\
R_{1}=\frac{r e^{-d_{1} \tau}}{\beta A_{1}^{2}+p_{1} A_{2}} .
\end{array}\right.
$$

Theorem 2. If $R_{1}<1$, the 'mature pest-extinction' periodic solution $\left(0, I^{*}(t), Y_{j}^{*}(t), Y^{*}(t)\right)$ of system (2.6) is globally attractive.

Proof: Let $\left(S(t), I(t), Y_{j}(t), Y(t)\right)$ be any solution of system (2.6) with the initial condition (2.4). From the second equation of system ()$, \frac{d I}{d t} \geq-\left(d_{3}+\right.$ $\left.d_{4}\right) I(t), I\left(t^{+}\right)=I(t)+p$ for $n T<t \leq(n+1) T$. By lemma (5) the impulsive differential equation (IDE)system:

$$
\left\{\begin{array}{l}
\frac{d z_{1}(t)}{d t}=-\left(d_{3}+d_{4}\right) z_{1}(t), t \neq n T \\
\triangle z_{1}(t)=p, t=n T
\end{array}\right.
$$

has a globally asymptotically stable positive solution, $z_{1}^{*}(t)=\frac{p e^{-\left(d_{3}+d_{4}\right)(t-n T)}}{1-e^{-\left(d_{3}+d_{4}\right) T}}, t \in$ $(n T,(n+1) T]$. Then for any sufficiently small $\varepsilon>0$, there exists an integer $n^{*}$ such that

$$
\frac{d I}{d t}>z_{1}^{*}(t)-\varepsilon>\frac{p e^{-\left(d_{3}+d_{4}\right) T}}{1-e^{-\left(d_{3}+d_{4}\right) T}}-\varepsilon=A_{1}-\varepsilon, t>n^{*} T .
$$


Similarly, for any sufficiently small $\varepsilon_{1}>0$, there exists an integer $n^{* *}>n^{*}$ such that,

$$
Y_{j}(t)>Y_{j}^{*}(t)-\varepsilon_{1}, t \in((n+l-1) T,(n+l) T], t>n^{* *} T,
$$

from this and equation (2.6), we have $\frac{d Y(t)}{d t} \geq m Y_{j}^{*}-d_{6} Y(t) \Rightarrow \frac{d Y(t)}{d t} \geq m\left(Y_{j}^{*}(t)-\right.$ $\left.\varepsilon_{1}\right)-d_{6} Y(t)$. Consider the comparison system

$$
\left\{\begin{array}{l}
\frac{d z_{2}(t)}{d t}=m\left(Y_{j}^{*}(t)-\varepsilon_{1}\right)-d_{6} z_{2}(t), t \neq(n+l-1) T \\
\triangle z_{2}(t)=0, t=(n+l-1) T
\end{array}\right.
$$

by the similar result of [26], we note that this system has a globally asymptotically stable positive periodic solution, $z_{2}^{*}(t)=g(t)-\frac{m \varepsilon_{1}}{d_{6}}, t \in((n+l-1) T,(n+$ l) $T]$, where

$$
g(t)=\frac{m q\left(\left(1-e^{-\left(m+d_{5} T\right)}\right) e^{-d_{6}(t-(n+l-1) T)}-\left(1-e^{-d_{6} T}\right) e^{-\left(m+d_{5}\right)(t-(n+l-1) T)}\right)}{\left(m+d_{5}-d_{6}\right)\left(1-e^{-\left(m+d_{5}\right) T}\right)\left(1-e^{d_{6} T}\right)}
$$

and $g(t)$ is continuous function on $t \in((n+l-1) T,(n+l) T]$. Further, it has a unique stationary point $t^{*}=(n+l-1) T+\frac{1}{m+d_{5}-d_{6}} \ln \frac{\left(m+d_{5}\right)\left(1-e^{-d_{6} T}\right)}{d_{6}\left(1-e^{-\left(m+d_{5}\right) T}\right)}$ at which double derivative $g^{\prime \prime}\left(t^{*}\right)=-m q \bigwedge<0$, where

$$
\bigwedge=\frac{\left.d_{6}^{\frac{m+d_{5}}{m+d_{5}-d_{6}}}\left(1-e^{(}-\left(m+d_{4}\right) T\right)\right)^{\frac{d_{6}}{m+d_{5}-d_{6}}}}{\left(m+d_{5}\right)^{\frac{d_{6}}{m+d_{5}-d_{6}}}\left(1-e^{-d_{6} T}\right)^{\frac{m+d_{5}}{m+d_{5}-d_{6}}}}
$$

now we see that $g\left(t^{*}\right)=\frac{m q\left(1-\frac{d_{6}}{\left(m+d_{5}\right)}\right)\left(\frac{d_{6}}{\left(m+d_{5}\right)}\left(1-e^{-\left(m+d_{5}\right) T}\right)^{\frac{d_{6}}{\left(m+d_{5}-d_{6}\right)}}\right)}{\left(m+d_{5}-d_{6}\right)\left(1-e^{-d_{6} T}\right)^{\frac{m+d_{5}}{\left(m+d_{5}-d_{6}\right)}}} g((n+l-$ 1) $T)=\frac{m q\left(e^{-d_{6} T}-e^{-\left(m+d_{5}\right) T}\right)}{\left(m+d_{5}-d_{6}\right)\left(1-e^{-d_{6} T}\right)\left(1-e^{-\left(m+d_{5}\right) T}\right)}$ and

$$
g((n+l) T)=\frac{m q\left(e^{-d_{6} T}-e^{-\left(m+d_{5}\right) T}\right)}{\left(m+d_{5}-d_{6}\right)\left(1-e^{-d_{6} T}\right)\left(1-e^{-\left(m+d_{5}\right) T}\right)} .
$$

Thus $g\left(t^{*}\right)=B, g((n+l-1) T)=A_{2}=g((n+l) T)$, from discussion above we have

$$
A_{2} \leq g(t) \leq g\left(t^{*}\right)=B
$$

so for arbitrary sufficiently small $\varepsilon_{2}>0$, there exists an integer $n^{* *}>n^{*}$ and $n>n^{* *}$ such that $Y(t)>z_{2}^{*}(t)-\varepsilon_{2}, t \in((n+l-1) T,(n+l) T], \Rightarrow$

$$
y(t)>A_{2}-\frac{m \varepsilon_{1}}{d_{6}}-\varepsilon_{2}
$$


by using above equation, equation (3.8) and first equation of system (2.6), we have,

$$
\begin{array}{r}
\frac{d S(t)}{d t} \leq r e^{-d_{1} \tau} S(t-\tau)-d_{2} S^{2}(t)-\beta S(t)\left(A_{1}-\varepsilon\right)^{2}-p_{1} S(t)\left(A_{2}-\frac{m \varepsilon_{1}}{d_{6}}-\varepsilon_{2}\right) \\
, t>n^{* *} T+\tau
\end{array}
$$

Now consider the following comparison theorem,

$$
\begin{array}{r}
\frac{d z_{3}(t)}{d t} \leq r e^{-d_{1} \tau} z_{3}(t-\tau)-d_{2} z_{3}^{2}(t)-\beta z_{3}(t)\left(A_{1}-\varepsilon\right)^{2}-p_{1} z_{3}(t)\left(A_{2}-\frac{m \varepsilon_{1}}{d_{6}}-\varepsilon_{2}\right) \\
t>n^{* *} T+\tau \quad(3.14)
\end{array}
$$

since $R_{1}=\frac{r e^{-d_{1} \tau}}{\beta A_{1}^{2}+p_{1} A_{2}}<1 \Rightarrow$

$$
r e^{-d_{1} \tau}<\beta A_{1}^{2}+p_{1} A_{2}
$$

We can choose arbitrary sufficiently small $\varepsilon>0, \varepsilon_{1}>0, \varepsilon_{2}>0$ such that

$$
r e^{-d_{1} T}<\beta\left(A_{1}-\varepsilon\right)^{2}+p_{1}\left(A_{2}-\frac{m \varepsilon_{1}}{d_{6}}-\varepsilon_{2}\right) .
$$

But lemma (4) supplies the information that $\lim _{t \rightarrow \infty}\left(z_{3}(t)\right)=0$. From equation (3.13) and by comparison theorem (3.14), we have, $S(t) \leq z_{3}(t)$ for sufficiently large $\mathrm{t}$ therefore $\lim _{t \rightarrow \infty} S(t)=0$. Hence for sufficiently small $\varepsilon_{3}>0$ and large $t>0$, we have $0<S(t)<\varepsilon_{3}$. As a generalization, we can state that $0<S(t)<\varepsilon_{3}$ for $t \geq 0$. The second equation of system $(2.6)$ provides $\frac{d I(t)}{d t}<$ $\left(\beta \varepsilon_{3}\left(A_{1}-\varepsilon\right)-d_{3}-d_{4}\right) I(t)$. Consider the following comparison system,

$$
\left\{\begin{array}{l}
\frac{d z_{4}}{d t}=\left(\beta \varepsilon_{3}\left(A_{1}-\varepsilon\right)-d_{3}-d_{4}\right) I(t), t \neq n T, \\
\triangle z_{4}(t)=p, t=n T .
\end{array}\right.
$$

By lemma (5), we see that this system has globally asymptotically positive periodic solution

$z_{4}^{*}=\frac{p e^{-\left(-\beta \varepsilon_{3}\left(A_{1}-\varepsilon\right)+d_{3}+d_{4}\right)(t-n T)}}{1-e^{-\left(-\beta \varepsilon_{3}\left(A_{1}-\varepsilon\right)+d_{3}+d_{4}\right)} T}$. Thus, for a sufficiently small $\varepsilon_{4}>0$ and $\mathrm{t}$ is sufficiently large, we have

$$
I(t)<z_{4}(t)<z_{4}^{*}(t)+\varepsilon_{4}
$$

hence we have

$$
z_{1}^{*}(t)-\varepsilon<I(t)<z_{4}(t)<z_{4}^{*}(t)+\varepsilon_{4}
$$


Let $\varepsilon_{3}, \varepsilon, \varepsilon_{4} \rightarrow 0$, above equation shows that $\lim _{t \rightarrow \infty} I(t)=I^{*}(t)$. Since, we have proved in lemmas (1-2) that our system has positive and bounded solution, therefore there exists a bound $\overline{Y(t)}$ of $Y(t)$, now from third equation of our system (2.6), we have

$$
\frac{d Y_{j}(t)}{d t} \leq q_{1} p_{1} \varepsilon_{3} \overline{Y(t)}-\left(m+d_{5}\right) Y_{j}(t)
$$

Consider the comparison system

$$
\left\{\begin{array}{l}
\frac{d z_{5}(t)}{d t}=q_{1} p_{1} \varepsilon_{3} \overline{Y(t)}-\left(m+d_{5}\right) z_{5}(t), t \neq(n+l-1) T \\
\triangle z_{5}(t)=q, t=(n+l-1) T
\end{array}\right.
$$

there exists a has globally asymptotically positive periodic solution for the above system

$$
z_{5}^{*}(t)=\frac{q_{1} p_{1} \varepsilon_{3} \overline{Y(t)}}{\left(m+d_{5}\right)}+\frac{q e^{-\left(m+d_{5}\right)(t-(n+l-1) T)}}{1-e^{\left(m+d_{5}\right) T}}, t \in((n+l-1) T,(n+l) T] \text { hence }
$$

for a sufficiently small $\varepsilon_{5}>0$ and large $t$ by comparison theorem, we have the following

$$
Y_{j}(t)<z_{5}(t)<z_{5}^{*}(t)+\varepsilon_{5}
$$

from the equation (3.9) and above, we have the following,

$$
Y_{j}^{*}(t)-\varepsilon_{1}<Y_{j}(t)<z_{5}(t)<z_{5}^{*}(t)+\varepsilon_{5}
$$

let $\varepsilon_{1}, \varepsilon_{3}, \varepsilon_{5} \rightarrow \rightarrow 0$ we have $\lim _{t \rightarrow \infty}\left(Y_{j}(t)\right)=Y_{j}^{*}(t)$. Since $\lim _{t \rightarrow \infty}\left(Y_{j}(t)\right)=$ $Y_{j}^{*}(t)$ by lemma $(6)$ we have $\lim _{t \rightarrow \infty}(Y(t))=Y^{*}(t)$. The proof is completed.

\section{Uniform Persistence and the Pest Control Strategy}

In previous section, we have proved that the 'mature pest-extinction' periodic solution $\left(0, I^{*}(t), Y_{j}^{*}(t), Y^{*}(t)\right)$ of system is globally attractive when $R_{1}<1$. In other words we say that the adult pest population is eradicated totally as time moves under the condition for the global attractivity. From ecological and biological point we want to control the pest population under some some level so called economic threshold level(ETL).

In this section we will prove the sufficient condition for the uniform persistence of our mathematical model. Further we will also discuss about the 
strategy of regulating the pest. Let us denote the following before stating our main theorems of this section.

$$
\left\{\begin{array}{l}
A=\frac{m p_{1} q_{1} L}{\left(m+d_{5}\right) d_{6}} \\
R_{2}=\frac{\left(r e^{-d_{1} \tau}-\frac{\beta p L}{1-e^{-\left(d_{3}+d_{4}\right) T}}\right)}{p_{1} B} \\
B_{1}=-\left(d_{2}+\frac{\beta^{2} L^{3}}{\left(d_{3}+d_{4}\right)}+p_{1} A\right) \\
k^{*}=\frac{\left(R_{2}-1\right) p_{1} B}{B_{1}}
\end{array}\right.
$$

Theorem 3. If $R_{2}>1$, then system (2.6) is uniform persistence (permanent).

Proof. Firstly, we will show that there exists a $\sigma$ such that $S(t) \geq \sigma$ for sufficiently large t. First equation of our system (2.6) gives

$$
\begin{aligned}
& \frac{d S}{d t}=\left[r e^{-d_{1} \tau}-d_{2} S-\beta I^{2}-p_{1} Y(t)\right] S(t)-r e^{-d_{1} \tau} \frac{d}{d t} \int_{t-\tau}^{t} S(\varrho) d \varrho . \\
& \text { Let } U(t)=S(t)+r e^{-d_{1} \tau} \frac{d}{d t} \int_{t-\tau}^{t} S(\varrho) d \varrho \\
& \Rightarrow \frac{d U(t)}{d t}=\frac{d S(t)}{d t}+r e^{-d_{1} \tau} \frac{d}{d t} \int_{t-\tau}^{t} S(\varrho) d \varrho \\
& \Rightarrow \frac{d U(t)}{d t}=\left[r e^{-d_{1} \tau}-d_{2} S-\beta I^{2}-p_{1} Y(t)\right] S(t)
\end{aligned}
$$

Next we claim that there exists no $k^{*}$ for all $t \geq t_{0}$ such that $S(t)<k^{*}$. We will prove it by contradiction method. Let this is true i.e. $S(t)<k^{*}$ for $t \geq t_{0}$. Now second equation of our system (2.6) will give us

$$
\left\{\begin{array}{l}
\frac{d I}{d t} \leq \beta k^{*} L^{2}-\left(d_{3}+d_{4}\right) I(t), t \neq n T \\
\triangle I(t)=p, t=n T
\end{array}\right.
$$

thus there exists a $T_{1}^{\prime}>t_{0}+\tau$ such that

$$
\begin{aligned}
& I(t)<\frac{\beta k^{*} L^{2}}{d_{3}+d_{4}}+\frac{p e^{-\left(d_{3}+d_{4}\right)(t-n T)}}{1-e^{-\left(d_{3}+d_{4}\right) T}}+\epsilon_{1}<\frac{\beta k^{*} L^{2}}{d_{3}+d_{4}}+\frac{p}{1-e^{-\left(d_{3}+d_{4}\right) T}}+\epsilon_{1} \\
& \Rightarrow I(t)<\frac{\beta k^{*} L^{2}}{d_{3}+d_{4}}+\frac{p}{1-e^{-\left(d_{3}+d_{4}\right) T}}+\epsilon_{1} \equiv \Phi(\text { say }) .
\end{aligned}
$$

Similarly, from third equation of our system (2.6), we have,

$$
\left\{\begin{array}{l}
\frac{d Y_{j}(t)}{d t} \leq q_{1} p_{1} k^{*} L-\left(d_{5}+m\right) Y_{j}(t), t \neq(n+l-1) T \\
\triangle Y_{j}(t)=q, t=(n+l-1) T
\end{array}\right.
$$

thus there exists a $T_{1}^{\prime \prime}>t_{0}+\tau$ such that

$$
Y_{j}(t)<\frac{q_{1} p_{1} k^{*} L}{m+d_{5}}+\frac{q e^{-\left(m+d_{5}\right)(t-(n+l-1) T)}}{1-e^{-\left(m+d_{5}\right) T}}+\epsilon_{2} \equiv \overline{Y_{j}}+\epsilon_{2} \text { (say). }
$$

Let $T_{1}=\max \left\{T_{1}^{\prime}, T_{1}^{\prime \prime}\right\}$ and fourth equation of our system (2.6), generates;

$$
\left\{\begin{array}{l}
\frac{d Y}{d t} \leq \overline{Y_{j}}+\epsilon_{2}-d_{6} Y(t), t \neq(n+l-1) T \\
\triangle Y(t)=0, t=(n+l-1) T
\end{array}\right.
$$


there exists a $T_{2}>T_{1}$ such that

$$
Y(t)<\frac{m}{d_{6}}\left(\frac{q_{1} p_{1} k^{*} L}{m+d_{5}}+\epsilon_{2}\right)+g(t)+\epsilon_{3}, t>T_{2}
$$

then from equation (3.11), we have,

$Y(t)<\frac{m}{d_{6}}\left(\frac{q_{1} p_{1} k^{*} L}{m+d_{5}}+\epsilon_{2}\right)+B+\epsilon_{3} \equiv \Phi^{\prime}($ say $), t>T_{2}$.

Now, we have the following inequality

$$
r e^{-d_{1} \tau}>d_{2} k^{*}+\beta \Phi L+p_{1} \Phi^{\prime}
$$

proof of this inequality can see at appendix. By the above inequality we have the following

$$
\frac{d U}{d t}>\left(r e^{-d_{1} \tau}-d_{2} k^{*}-\beta \Phi L-p_{1} \Phi^{\prime}\right) S(l), t>\widetilde{T} .
$$

Let $S^{l}=\min \left[T_{1}, T_{1}+\tau\right] S(l)$. We can show that $S(t) \geq S^{l}$ for all $t \geq \widetilde{T}$. In this direction, there exists a non-negative constant $T_{3}$ such that $S(t) \geq S^{l}$ for all $t \geq\left[\widetilde{T}, \widetilde{T}+\tau+T_{3}\right]$

$S\left(\widetilde{T}, \widetilde{T}+\tau+T_{3}\right)=S^{l}$ and

$\left.\frac{d S}{d t}\right|_{\mathbf{P}_{+\tau+T_{3}}} \leq 0$. Therefore, we have

$\frac{d S\left(\boldsymbol{e}+\tau+T_{3}\right)}{d t}>\left(r e^{-d_{1} \tau}-d_{2} k^{*}-\beta \Phi L-p_{1} \Phi^{\prime}\right) S^{l}>0$

thus, we arrive at a contradiction. Hence $S(l) \geq S^{l}>\underset{\sim}{0}$ and

$\frac{d U}{d t}>\left(r e^{-d_{1} \tau}-d_{2} k^{*}-\beta \Phi L-p_{1} \Phi^{\prime}\right) S^{l}>0$ for all $t \geq \widetilde{T}$.

From this we have $U(t) \rightarrow \infty$ as $t \rightarrow \infty$ which is a contradiction to $U(t) \leq$ $\left(1+r \tau e^{-d_{1} \tau}\right) L$, therefore for any constant $t_{0}>0$ the condition $S(t)<k^{*}$ can not hold for all $t \geq t_{0}$.

Let $\sigma=\min \left\{\frac{1}{2} k^{*}, k^{*} \exp ^{-\left(d_{2} L+\beta L^{2}+p_{1} L\right) \tau}\right\}$. Now, we shall prove that $S(t) \geq$ $\sigma$. For this, let us assume that there exists two positive constants $\bar{t}$ and $\rho$ such that $S(\bar{t})=S(\bar{t}+\rho)=k^{*}$ and $S(t)<k^{*}$ for $\bar{t}<t<\bar{t}+\rho$.

$$
\begin{aligned}
& \frac{d Y_{j}}{d t} \leq q_{1} p_{1} k^{*} L-\left(m+d_{5}\right) Y_{j}, t \neq(n+l-1) T \\
& \triangle Y_{j}=q, t=(n+l-1) T
\end{aligned}
$$

thus there exists a $T_{1}>\bar{t}+\tau$ such that

$$
Y_{j}(t)<\frac{q_{1} p_{1} k^{*} L}{m+d_{5}}+\frac{q e^{-\left(m+d_{5}\right)(t-(n+l-1) T)}}{1-e^{-\left(m+d_{5}\right) T}}+\epsilon_{2} \equiv \overline{Y_{j}}+\epsilon_{2}, t>T_{1} \text { (say). }
$$

Again fourth equation of the system (), we have, $\frac{d Y}{d t} \leq \overline{Y_{j}}+\epsilon_{2}-d_{6} Y(t), t \neq$ $(n+l-1) T$

$$
\triangle Y(t)=0, t=(n+l-1) T
$$

there exists a $T_{2}>T_{1}$ such that

$$
Y(t)<\frac{m}{d_{6}}\left(\frac{q_{1} p_{1} k^{*} L}{m+d_{5}}+\epsilon_{2}\right)+g(t)+\epsilon_{3}, t>T_{2}
$$

then, from equation we have

$$
Y(t)<\frac{m}{d_{6}}\left(\frac{q_{1} p_{1} k^{*} L}{m+d_{5}}+\epsilon_{2}\right)+B+\epsilon_{3} \equiv \Phi^{\prime}(\text { say }), \tilde{t}+T_{2}<t<\tilde{t}+\rho .
$$

Since $S(t)$ is bounded and continuous and impulse have on effect on it, we conclude that $S(t)$ is uniformly continuous. Hence there exists a constant $T_{4}$ such that $0<T_{4}<\tau$ and is independent of $\widetilde{t}$ such that $S(t)>\frac{1}{2} k^{*}$ for all 
$\tilde{t}<t<\tilde{t}+T_{4}$. If $\rho \leq T_{4}$ then $S(t) \leq \rho$ is obtained. Now we can discuss different cases: If $T_{4}<\rho \leq \tau$, from first equation of our system we have

$$
\frac{d S}{d t} \geq-\left(d_{2} L+\beta L^{2}+p_{1} L\right) S(t), \bar{t}<t \leq \bar{t}+\rho .
$$

Then we have,

$$
\begin{aligned}
& S(t) \geq e^{-\left(d_{2} L+\beta L^{2}+p_{1} L\right) \tau} S(\bar{t}) \text { or } \\
& S(t) \geq e^{-\left(d_{2} L+\beta L^{2}+p_{1} L\right) \tau} k^{*} .
\end{aligned}
$$

Then, we have $S(t) \leq \rho$ for $\bar{t}<t \leq \bar{t}+\rho$.

Now if $\rho \geq \tau$, then we have

$$
S(t) \geq e^{-\left(d_{2} L+\beta L^{2}+p_{1} L\right) \tau} k^{*} \text { for } \bar{t}<t \leq \bar{t}+\tau \text {. }
$$

Now we will show that $S(t) \geq e^{-\left(d_{2} L+\overline{\beta L}{ }^{2}+p_{1} L\right) \tau} k^{*}$ for $\bar{t}+\tau<t<\bar{t}+\rho$. We will prove it by the method of contraction. Suppose this is not true, then there exists a $T_{5}>0$ such that $S(t) \geq e^{-\left(d_{2} L+\beta L^{2}+p_{1} L\right) \tau} k^{*}$ for $\bar{t}<t \leq \bar{t}+\tau+T_{5}$ i.e. $S\left(\bar{t}+\tau+T_{5}\right)=k^{*} e^{-\left(d_{2} L+\beta L^{2}+p_{1} L\right) \tau}$ and $\frac{d S\left(\bar{t}+\tau+T_{5}\right)}{d t} \leq 0$.

But from second equation of (2.6) $\frac{d S\left(\bar{t}+\tau+T_{5}\right)}{d t}>\left(r e^{-d_{1} \tau}-d_{2} k^{*}-\beta \Phi L-p_{1} \Phi^{\prime}\right) S^{l}>$ 0

which is a contradiction to $\frac{d S\left(\bar{t}+\tau+T_{5}\right)}{d t} \leq 0$.

Hence we are now in position to conclude $S(t) \geq \sigma>0$ for all $t \in[\bar{t}, \bar{t}+\sigma]$. Since $[\bar{t}, \bar{t}+\sigma]$ is arbitrary hence $S(t) \geq \rho$ for sufficiently large t. Hence $S(t) \geq \sigma$ for all $t>T^{*}$.

Now we proceed for permanence of the system. Suppose that $(S(t), I(t))$, $\left.Y_{j}(t), Y(t)\right)$ is any positive solution of system (2.6) with initial conditions (2.4). From the proof of theorem (2), we have the following inequalities:

$$
\left\{\begin{array}{l}
I(t)>A_{1}-\epsilon_{1}, \\
Y_{j}(t)>\frac{q e^{-\left(m+d_{5}\right) T}}{1-e^{-\left(m+d_{5}\right) T}}-\epsilon_{1}, \\
Y(t)>A_{2}-\frac{m \epsilon_{2}}{d_{6}}-\epsilon_{2},
\end{array}\right.
$$

for sufficiently large t. By the boundedness of the solution $\left.(S(t), I(t)), Y_{j}(t), Y(t)\right)$ we can conclude the theorem.

When $R_{2}=1$, we can work out critical(threshold) values of parameters $\mathrm{p}$, q and $\tau$ respectively.

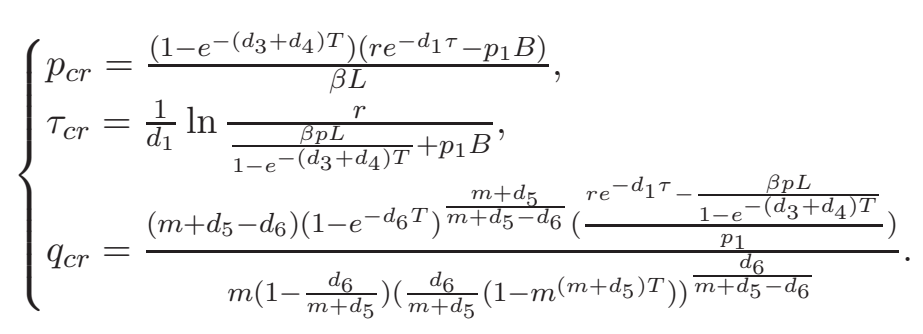


If our system (2.3) has the values of $p, q$ and $\tau$ less than critical values $p_{c r}, q_{c r}, \tau_{c r}$ respectively then our system is permanent.

As discussed in the introduction part that our aim in pest control problem to keep pests under the economic threshold level(ETL) to protect the crop. In the next theorem we prove the conditions under which the pest population is under ETL.

Theorem 4. For our system (2.6), let the following inequality $r e^{-d_{1} \tau}-d_{2} E<\beta A_{1}^{2}+p_{1} A_{2}<r e^{-d_{1} \tau}$

holds, then the pest and its natural enemy may coexist. Further, when $t$ is sufficiently large, we have, we have $S(t)<E$, where the constant $E$ is ETL.

Proof Suppose $\left(S(t), I(t), Y_{j}(t), Y(t)\right)$ is a positive solution of our system (2.6) with initial conditions (2.4). Since

$\beta A_{1}^{2}+\alpha A_{2}<r e^{-d_{1} \tau}$

we may choose three sufficiently small positive constants $\epsilon, \epsilon_{1}, \epsilon_{2}$ such that

$$
r e^{-d_{1} \tau}>\beta\left(A_{1}-\epsilon\right)^{2}+p_{1}\left(p_{1} A_{2}-m \varepsilon_{1} / d_{6}-\varepsilon_{2}\right) .
$$

Further, the inequality

$$
r e^{-d_{1} \tau}-d_{2} E<\beta A_{1}^{2}+p_{1} A_{2}
$$

will generate,

$$
\frac{r e^{-d_{1} \tau}-\left(\beta\left(A_{1}-\epsilon\right)^{2}+p_{1} A_{2}-m \varepsilon_{1} / d_{6}-\varepsilon_{2}\right)}{d_{2}}<E
$$

when $\mathrm{t}$ is sufficiently large then by equation (3.13) we have

$$
\frac{d S(t)}{d t} \leq r e^{-d_{1} \tau} S(t-\tau)-d_{2} S^{2}(t)-\left(\beta\left(A_{1}-\epsilon\right)^{2}+p_{1} A_{2}-m \varepsilon_{1} / d_{6}-\varepsilon_{2}\right) S(t) .
$$

By comparison theorem $\frac{d Z(t)}{d t} \leq r e^{-d_{1} \tau} Z(t-\tau)-d_{2} Z^{2}(t)-\left(\beta\left(A_{1}-\epsilon\right)^{2}+p_{1} A_{2}-\right.$ $\left.m \varepsilon_{1} / d_{6}-\varepsilon_{2}\right) Z(t)$.

Using lemma (4), we get,

$$
\begin{aligned}
& \lim _{t \rightarrow+\infty} Z(t)=\frac{r e^{-d_{1} \tau}-\left(\beta\left(A_{1}-\epsilon\right)^{2}+p_{1} A_{2}-m \varepsilon_{1} / d_{6}-\varepsilon_{2}\right)}{d_{2}} \\
& \Rightarrow \\
& \lim \sup _{t \rightarrow+\infty} S(t) \leq \frac{r e^{-d_{1} \tau}-\left(\beta\left(A_{1}-\epsilon\right)^{2}+p_{1} A_{2}-m \varepsilon_{1} / d_{6}-\varepsilon_{2}\right)}{d_{2}}<E
\end{aligned}
$$

proof is completed.

The E calculated above is very important in the pest control problems. This is the level upto which the pest may be used.

\section{Discussion}

In this paper, we modify the model of [26] by incorporating linear functional response, which makes the model more simple and practical from biological 
and ecological point of view. The results for boundedness, positivity, global attractivity, uniform persistence, economic threshold level (ETL) of the system (2.3) and (2.6) have been discussed in the text.

In Section 2, we have showed that our system (2.3) is dissipative and derived the conditions for which the system is uniform persistent. Uniform persistence concerns long term survival of the species so considered. Indeed, for a dissipative system, uniform persistence is somehow equivalent to the permanence. In Section3, global attractivity of mature prey extinction periodic solution is also obtained (Ref. Th.1 and 2). ETL is obtained in Section 4. ETL has many applications in the pest control strategy. It also important to mention that this study is an abstract study and is not a case study, hence the real parameters are not available. However, real parameter investigation is left for future work.

Before ending this section, we would like to share that there is a possibility for improvement of the current model (2.3). We propose the following model with a generalized functional response which is more practical and realistic as compared to the model (2.3);

$$
\begin{cases}\frac{d S_{j}(t)}{d t}=r S(t)-d_{1} S_{j}(t)-r e^{-d_{1} \tau} S(t-\tau), & t \neq(n+l-1) T, t \neq n T, \\ \frac{d S(t)}{d t}=r e^{-d_{1} \tau} S(t-\tau)-d_{2} S^{2}(t)-\beta S(t) I^{2}(t) & \\ -\frac{p_{1} S(t)^{2}}{\alpha S(t)^{2}+\beta S(t) Y(t)+Y(t)^{2}}, & t \neq(n+l-1) T, t \neq n T, \\ \frac{d I(t)}{d t}=\beta S(t) I^{2}(t)-\left(d_{3}+d_{4}\right) I(t), & t \neq(n+l-1) T, t \neq n T, \\ \frac{d Y_{j}(t)}{d t}=\frac{q_{1} p_{1} S(t)^{2}}{\alpha S(t)^{2}+\beta S(t) Y(t)+Y(t)^{2}}-\left(m+d_{5}\right) Y_{j}(t), & t \neq(n+l-1) T, t \neq n T, \\ \frac{d Y(t)}{d t}=m Y_{j}(t)-d_{6} Y(t), & t \neq(n+l-1) T, t \neq n T, \\ \Delta S_{j}(t)=0, \Delta S(t)=0, \Delta I(t)=0, & t=(n+l-1) T, \\ \Delta Y_{j}(t)=q, \Delta Y(t)=0, & t=n T, \\ \Delta S_{j}(t)=0, \Delta S(t)=0, \Delta I(t)=p, & \\ \Delta Y_{j}(t)=0, \Delta Y(t)=0, & \end{cases}
$$

where, $\alpha$ and $\beta$ are new constants. We think that the model system (5.1) will cause the analysis much richer and interesting dynamics. We keep it for our further future work.

Acknowledgements: We are grateful to Prof. Edoardo Beretta, Instituto do Biomatematica, Universita di Urbino, I-61029, Urbino, Italy for valuable suggestions for preparation of this manuscript.

\section{Appendix}

The proof of inequality (4.2) We will prove on the same line as in [26]. Let $k^{* *}$ satisfying our claimed inequality, then we have, 


$$
r e^{-d_{1} \tau}>d_{2} k^{* *}+\beta \Phi L+p_{1} \Phi^{\prime}
$$

Now put the values of $\Phi$ and $\Phi^{\prime}$, we have

$$
\begin{aligned}
r e^{-d_{1} \tau}>d_{2} k^{* *}+\beta\left(\frac{\beta k^{* *} L^{2}}{d_{3}+d_{4}}+\frac{p}{1-e^{-\left(d_{3}+d_{4}\right) T}}+\epsilon_{1}\right) L \\
\quad+p_{1}\left(\frac{m}{d_{6}}\left(\frac{q_{1} p_{1} k^{* *} L}{m+d_{5}}+\epsilon_{2}\right)+B+\epsilon_{3}\right) .
\end{aligned}
$$

Now let $\epsilon_{1}, \epsilon_{2}, \epsilon_{3} \rightarrow 0$ in the above equation, we get

$$
r e^{-d_{1} \tau}>d_{2} k^{* *}+\beta\left(\frac{\beta k^{* *} L^{2}}{d_{3}+d_{4}}+\frac{p}{1-e^{-\left(d_{3}+d_{4}\right) T}}\right) L+p_{1}\left(\frac{m}{d_{6}}\left(\frac{q_{1} p_{1} k^{* *} L}{m+d_{5}}\right)+B\right)
$$

Simplifying our claimed inequality we have

$$
\left(r e^{-d_{1} \tau}-\frac{L p_{1} B}{1-e^{-\left(d_{3}+d_{4}\right) T}}\right)-p_{1} B=k^{* *}\left(d_{2}+\frac{L^{3} \beta^{2}}{\left(d_{3}+d_{4}\right)}+\frac{p_{1} m q_{1} p_{1} L}{d_{6}\left(m+d_{5}\right)}\right) .
$$

Recall that $\left(r e^{-d_{1} \tau}-\frac{L p_{1} B}{1-e^{-\left(d_{3}+d_{4}\right) T}}\right)=p_{1} B, B_{1}=-\left(d_{2}+\frac{L^{3} \beta^{2}}{\left(d_{3}+d_{4}\right)}+\frac{p_{1} m q_{1} p_{1} L}{d_{6}\left(m+d_{5}\right)}\right)$ $\left(R_{2}-1\right) p_{1} B+k^{* *} B_{1}<0$

from the above discussion it is proved that inequality is satisfied for any $k^{* *}$. This completes the proof.

\section{References}

[1] Bing, L., Lansun, C., Yujuang, Z., The effects of impulsive toxicant input on a population in a polluted environment, J. Biol. Syst., 11(3) (2003), 265 - 274. doi: 10.1142/S0218339003000907

[2] Bainov, D., Simeonov , P., System with impulsive effects: Stability, theory and applications, John Wiley and Sons, New York (1989).

[3] Chao, L., Zhang, Q., Zhang, X., Xiaodong, D., Dynamical behaviour in a stage-structured differential-algebriac prey-predator model with discreate time delay and harvesting, J. Comp. Appl. Math., 231(2) (2009), 612625. doi: 10.1016/j.cam.2009.04.011

[4] Chattopadhyay, J. , Arino, O., A predator-prey model with disease in prey, Non Linear Analysis, 36 (1999), 747 - 766. 
[5] Cui, J., Song, X., Permanence of predator-prey system with stage structure, Discrete Contin. Dyn. Syst. Ser. B, 4(3)(2004),547-554. doi: 10.3934/dcdsb.2004.4.547-554

[6] David, G., Haque, M., A predator-prey model with disease in the prey species only, Math. Methods Appl. Sci., 30(8)(2007), 911 - 929. doi: $10.1002 / \mathrm{mma} .815$

[7] Guang -Ping, H., Li, X.-L., Stability and Hopf bifurcation for a delayed predator-prey model with disease in the prey, Chaos, Solitons and Fractals 45 (2012), 229 - 237. doi: 10.1016/j.chaos.2011.11.011.

[8] Gourley, S.A., Kuang, Y., A stage structureed predator-prey model and its dependence on maturation delay and death rate, J. Math. Bio. 49(2)(2004),188-200. doi: 10.1007/soo285-004-0278-2

[9] Jian-Jun-Jiao, Permananece and global attractivity of stage-structured predator-prey model with continous harvesting on predator and impulsive stocking on prey, Appl. Math. Mech.(Engl. Ed.), 29(5)(2008), 653-663. doi: 10.1007/S10483-008-0509-X.

[10] Kermack, W.O., Mckendrick , A.G., Contributons to the mathematical Theory of Epidimics, Part 1, Proc. Roy. Soc. A, 115(5)(1927), 700-721.

[11] Litao, H., Zhien, M., Hethcote, H.W., Four Predator Prey Models with Infectious Diseases, Mathematical and Computer Modeling, 34(7-8)(2001), 849 - 858. doi: 10.1016/S0895-7177(01)00104-2.

[12] Linfei, N., Zhidong, T., Singular perturbation method for global stability of ratio dependent predator-prey model with stage structure for the prey, Electronic Journal of Differential Equations, $\mathbf{8 6}(2013), \quad 1-9 . \quad$ http://ejde.math.txstate.edu or http://ejde.math.unt.eduftpejde.math.txstate.edu.

[13] Lakshmikantham, V. , Bainov, D., Simeonov, P., Theory of Implulsive Differential Equations, World Scientific, Singapore (1989).

[14] Meng, X., Chen, L., A stage structured SI eco-epidemiological model with time dealy and implusive controlling, J. Syst. Sci. Complex, 21(3)(2008),427-440. doi: 10.1007/S11424-008-9214-8 
[15] Maoxing, L., Zhen, J., Mainul, H., An Impulsive predator-prey model with communicable disease in the prey species only, Non Linear analysis: Real World Applications, 10(2009), 3098 - 3111. doi: 10.1016/j.nonrwa.2008.10.010.

[16] Mukhopadhyaya, B., Bhattacharyya, B., Dynamics of a delay-diffusion prey-predator Model with disease in the prey, J. Appl. Math and Computing, 17(12)(2005), 361 - 377. doi: 10.1007/BF02936062

[17] Mainul, H., Venturino, E., Increase of the prey may decrease the healthy prtedator population in presence of a disease in predator, HERMIS, 7(2006), 38-59. www.aueb.br/pympe/hermis-volume-7/DIFFINTEGRAL-EQUATIONS/HAQUE-VENTURINO-1.pdf

[18] Mathur, H. B., Agarwal, H. C., Johnson, S., Saikia, N., Analysis of Pesticide Residues in Blood Samples from Villages of Punjab, Centre for Science and Environment, New Delhi, 1 - 24(2005)(Accessed on 20 October, 2014) http://indiaenvironmentportal.org.in/files/Punjab_blood_report.pdf

[19] Paul G., Hsieh, Y.-H., Global dynamics of a predator-prey model with stage-structure for the predator, SIAM J. Appl. Math., 67(5) (2007),13791395. doi: 10.1137/06070377.

[20] Raid, K. N., Kawa, A.H., The dynamics of prey-predator model with disease in the prey, J. Math. Comput. Sci., 2 (4) (2012), 1052-1072.

[21] Rui, X., Chaplain, M.A.J., Davidson, F.A., Periodic solutions of a delayed predator prey model with stage structure for predator, J. Appl. Math., 3(2004), 255-270. doi: 10.1155/S1110757X04308090

[22] Samanta, G.P., Analysis of a delay nonautonomous predator-prey system with disease in the prey, Non Linear Analysis: Modeling and Control, 15(1) (2010), 97-108. www.lana.lt/journal/36/samanta.pdf

[23] Soovoojeet, J., Kar, T.K., Modeling and Analysis of a prey-predator system with disease in the prey, Chaos, Solitons and Fractals 47(2013), 42-53. doi: $10.1016 /$ j.chaos.2012.12.002

[24] Shengqiung, L., Edoardo, B., A stage-structured predator-prey model of Beddington-DeAgelis type, SIAM J. Appl. Math., 66(4)(2006),11011129. doi: 10.1137/050630003. 
[25] Song, X., Chen, L., Optimal harvesting and stability for a two species competitive system with stage structure, Math. Biosci., 170(2)(2001), 173-186. doi: 10.1016/S0025-5564(00)00068-7

[26] Tongqian, Z., Meng, X., Song, Y., Zhang, T., A stage-structured predator-prey SI model with disease in the prey and impulsive effetcs, Mathematical Modelling and Analysis, 18(4)(2013), 505-528. doi: 10.3846/13926292.2013.840866.

[27] Volterra, V., Variazioni e fluttauazionidel numero d individui in species animals conviventii, Mem Acd. Linciei, 2(1926), 31 - 113.

[28] Wang, W., Chen, L., A predator-prey system with age structure for predator, Comp. Math. Appl. 33(8) (1997), 83-91. doi: 10.1016/So8981221(97)00056-4

[29] Xu, R., Chaplain, M.A.J., Davidson, F.A., Perisistence and global stability of a ratio dependent prey model with stage-structure, Appl. Math. Comp., 158(3) (2004), 729-744. doi: 10.1016/j.amc.2003.10.012

[30] Xiaohong, T., Rui, X., Global dynamics of a predator-prey system with Holling type II functional response, Nonlinear Analysis: Modelling and Control, 16(2)(2011), 242-253. www.lana.lt/journal/41/NA16210.pdf

[31] Xue, Z., Zhang, Q.-L., Chao, L., Xiang, Z.-Y., Bifurcations of a singular prey-predator economic model with time delay and stage structure, Chaos Soliton and Fractals, 42 (2009), 1485-1494. doi: 10.1016/j.chaos.2009.03.051

[32] Xinyu, S., Meiying, H., Xinzhu, M., A stage-structureed predator-prey model with disturbing pulse and time delays, Appl. Math. Modell., 33(1) (2009), 211-223. doi: 10.1016/j.apm.2007.10.020

[33] Yanni, X., Lansun, C., Modeling and analysis of a predator-prey model with disease in the prey, Math. Biosci., 171(1) (2001), 59-82. doi: 10.1016/S0025-5534(01)00049-9

[34] Yonghui, X., Jinde, C., Sui, S.C., Multiple periodic solutions of a delayed stage-structured predator-prey model with non-monotonic functional responses, Appl. Math. Modell., 31(9)(2007), 1947-1959. doi: 10.1016/j.apm.2006.08.012 
[35] Zhongui, X., A Lotka-Volterra model with impulsive effects on the prey and stage structure on the predator, Appl. Math. Sci., 4(17-20) (2010), 849856. www.m-hikari.com/ams/ams-2010/ams-17-20-2010/xiangAMS17-202010.pdf

[36] Zhang, T., Meng, X., Song, Y., The dynamics of a high dimensional delayed pest management model with impulsive pesticide input and harvesting prey at different fixed moments, Nonlinear Dynamics, 64(1)(2011), 1-12. doi: $10.1007 / \mathrm{s} 11071-010-9840-1$. 